\title{
Planting Kurepa trees and killing Jech-Kunen trees in a model by using one inaccessible cardinal
}

\author{
by
}

Saharon Shelah (Jerusalem and New Brunswick, N.J.) and Renling J in (Berkeley, Calif.)

\begin{abstract}
By an $\omega_{1}$-tree we mean a tree of power $\omega_{1}$ and height $\omega_{1}$. Under $\mathrm{CH}$ and $2^{\omega_{1}}>\omega_{2}$ we call an $\omega_{1}$-tree a Jech-Kunen tree if it has $\kappa$-many branches for some $\kappa$ strictly between $\omega_{1}$ and $2^{\omega_{1}}$. In this paper we prove that, assuming the existence of one inaccessible cardinal, (1) it is consistent with $\mathrm{CH}$ plus $2^{\omega_{1}}>\omega_{2}$ that there exist Kurepa trees and there are no Jech-Kunen trees, which answers a question of [Ji2], (2) it is consistent with $\mathrm{CH}$ plus $2^{\omega_{1}}=\omega_{4}$ that there only exist Kurepa trees with $\omega_{3}$-many branches, which answers another question of [Ji2].
\end{abstract}

A partially ordered set, or poset for short, $\left\langle T,<_{T}\right\rangle$ is called a tree if for every $t \in T$ the set $\left\{s \in T: s<_{T} t\right\}$ is well-ordered under $<_{T}$. The order type of that set is called the height of $t$ in $T$, denoted by ht $(t)$. We will not distinguish a tree from its base set. For every ordinal $\alpha$, let $T_{\alpha}$, the $\alpha$-th level of $T$, be $\{t \in T: \operatorname{ht}(t)=\alpha\}$ and $T\left\lceil\alpha=\bigcup_{\beta<\alpha} T_{\beta}\right.$. Let ht $(T)$, the height of $T$, be the smallest ordinal $\alpha$ such that $T_{\alpha}=\emptyset$. By a branch of $T$ we mean a linearly ordered subset of $T$ which intersects every nonempty level of $T$. Let $\mathcal{B}(T)$ be the set of all branches of $T . T^{\prime}$ is called a subtree of $T$ if $T^{\prime} \subseteq T$, $<_{T^{\prime}}=<_{T} \cap T^{\prime} \times T^{\prime}\left(T^{\prime}\right.$ inherits the order of $\left.T\right)$ and for every $\alpha<\operatorname{ht}\left(T^{\prime}\right)$, $T_{\alpha}^{\prime} \subseteq T_{\alpha}$.

$T$ is called an $\omega_{1}$-tree if $|T|=\omega_{1}$ and $\operatorname{ht}(T)=\omega_{1}$. An $\omega_{1}$-tree $T$ is called a Kurepa tree if $|\mathcal{B}(T)|>\omega_{1}$ and for every $\alpha \in \omega_{1},\left|T_{\alpha}\right|<\omega_{1}$. An $\omega_{1}$-tree is called a Jech-Kunen tree if $\omega_{1}<|\mathcal{B}(T)|<2^{\omega_{1}}$.

T. Jech in [Je1] constructed by forcing a model of CH plus $2^{\omega_{1}}>\omega_{2}$ in which there is a Jech-Kunen tree. In fact, it is a Kurepa tree with fewer than $2^{\omega_{1}}$-many branches. Late, K. Kunen [K1] found a model of CH plus $2^{\omega_{1}}>$

1991 Mathematics Subject Classification: Primary 03E35.

The research of the first author was partially supported by the United States-Israel Binational Science Foundation, publ. 469. 
$\omega_{2}$ in which there are neither Kurepa trees nor Jech-Kunen trees. In his paper he gave an equivalent form of Jech-Kunen trees in terms of compact Hausdorff spaces. The detailed proof can be found in [Ju, Theorem 4.8].

The second author in [Ji1] started discussing the differences between the existence of Kurepa trees and the existence of Jech-Kunen trees. He showed that it is independent of $\mathrm{CH}$ plus $2^{\omega_{1}}>\omega_{2}$ that (1) there exists a Kurepa tree which has no Jech-Kunen subtrees, (2) there exists a JechKunen tree which has no Kurepa subtrees. In his proofs some strongly inaccessible cardinals were assumed to exist and later, Kunen eliminated the large cardinal assumption from one of the proofs.

In [SJ], we answered a question of [Ji2] by proving that, assuming the existence of one inaccessible cardinal, it is consistent with $\mathrm{CH}$ plus $2^{\omega_{1}}>\omega_{2}$ that there exist Jech-Kunen trees and there are no Kurepa trees.

In [Ji2], the second author proved that, assuming the existence of two inaccessible cardinals, it is consistent with $\mathrm{CH}$ plus $2^{\omega_{1}}>\omega_{2}$ that there exist Kurepa trees and there are no Jech-Kunen trees.

Since the consistency of the nonexistence of Jech-Kunen trees implies the consistency of the existence of an inaccessible cardinal [Ju, Theorem 4.10], we have to use at least one inaccessible cardinal to build a model of $\mathrm{CH}$ plus $2^{\omega_{1}}>\omega_{2}$ in which there are Kurepa trees but there are no Jech-Kunen trees. The question whether one inaccessible cardinal is enough was asked in [Ji2]. In this paper, we will give a positive answer to the question. We also discover that the same techniques can be used to answer another question in [Ji2] by constructing a model of $\mathrm{CH}$ plus $2^{\omega_{1}}=\omega_{4}$ in which there only exist Kurepa trees with $\omega_{3}$-many branches.

First let us look at the second author's original idea in [Ji2] to construct a model of CH plus $2^{\omega_{1}}>\omega_{2}$ in which there are Kurepa trees and there are no Jech-Kunen trees, by using two inaccessible cardinals. Let $\kappa_{1}<\kappa_{2}$ be two strongly inaccessible cardinals in a model $M$. First, Jin collapses $\kappa_{2}$ down to $\kappa_{1}^{+}$by forcing with a $<\kappa_{1}$-support Lévy collapsing order. Next, he collapses $\kappa_{1}$ down to $\omega_{1}$ by forcing with a finite support Lévy collapsing order. This step will create a very homogeneous Kurepa tree. Then he forces with that Kurepa tree $\lambda$-many times for some regular cardinal $\lambda$ which is greater than $\kappa_{2}$. In the resulting model, that Kurepa tree has $\lambda$-many branches and $\lambda=2^{\omega_{1}}$. In that model there are no Jech-Kunen trees.

If we want to obtain the same result by using only one inaccessible cardinal, we may have to find a way to create a homogeneous $\omega_{1}$-tree with every level countable, without the assistance of large cardinals. Then the following questions arise. First, how can we create the desired tree? Second, can we force with that tree many times (with countable supports) without collapsing $\omega_{1}$ ? (Note that a normal $\omega_{1}$-tree with every level countable is never $\omega_{1}$-closed.) 
In this paper, we construct a homogeneous generic $\omega_{1}$-tree with every level countable by forcing with an $\omega_{1}$-closed poset whose elements are countable homogeneous normal subtrees of $\left\langle 2^{\omega_{1}}, \subseteq\right.$. The generic tree is, in fact, a Suslin tree. Then we force with that generic tree $\lambda$-many times with countable supports. We will prove that this two-step forcing adds no new countable sequences of ordinals, hence it will not collapse $\omega_{1}$. We will also prove that if the ground model is Silver's model (see [K2, p. 259]), then in the final model there are no Jech-Kunen trees.

Before proving our results we need more notations and definitions.

A tree $T$ is called normal if

(1) every $t \in T$ which is not in the top level of $T$ has at least two immediate successors,

(2) for every limit ordinal $\alpha<\operatorname{ht}(T)$ and every $B \in \mathcal{B}(T\lceil\alpha)$, there exists at most one least upper bound of $B$ in $T$,

(3) for every $t \in T$ and $\alpha$ such that $\operatorname{ht}(t)<\alpha<\operatorname{ht}(T)$, there exists $t^{\prime} \in T_{\alpha}$ such that $t<_{T} t^{\prime}$.

Without loss of generality, we only consider the trees which are subtrees of $\left\langle 2^{<\omega_{1}}, \subseteq\right.$ with the unique root $\emptyset$. Let $T$ be a tree and $B \subseteq T$ be a totally ordered subset of $T$. Then $\cup B$ is the only candidate for the least upper bound of $B$ in $T$.

Let $\alpha \in \omega_{1}$ and $s, t \in 2^{\alpha}$. We define a map $F_{s, t}$ from $2^{<\omega_{1}}$ to $2^{<\omega_{1}}$. Let $u \in 2^{\beta}$ for some $\beta<\omega_{1}$. The domain of $F_{s, t}(u)$ is $\beta$ and for every $\gamma<\beta$, if $\gamma<\alpha$, then let

$$
F_{s, t}(u)(\gamma)=u(\gamma)+t(\gamma)-s(\gamma)(\bmod 2),
$$

otherwise let $F_{s, t}(u)(\gamma)=u(\gamma)$.

LEMMA 1. $F_{s, t}(s)=t, F_{s, t}(t)=s$ and $F_{s, t}$ is an isomorphism from $\left\langle 2^{<\beta}, \subseteq\right.$ to $\left\langle 2^{<\beta}, \subseteq\right.$ for any $\beta \leq \omega_{1}$.

\section{Pr o of. Trivial.}

A normal tree $T$ is called homogeneous if for any $\alpha<\operatorname{ht}(T)$ and any $s, t \in T_{\alpha}, F_{s, t} \uparrow T$ is an isomorphism from $T$ to $T$.

Let

$\mathbb{P}_{\text {hom }}=\left\{T: T\right.$ is a countable homogeneous normal subtree of $\left\langle 2^{\omega_{1}}, \subseteq\right\}$

be a poset ordered by letting

$$
T<T^{\prime} \quad \text { iff } \quad \operatorname{ht}\left(T^{\prime}\right)<\operatorname{ht}(T) \quad \text { and } \quad T^{\prime}=T \uparrow \operatorname{ht}\left(T^{\prime}\right) .
$$

Lemma 2. Let $\mathcal{T}$ be a totally ordered subset of $\mathbb{P}_{\text {hom }}$. Then $\bigcup \mathcal{T}$ is a homogeneous tree. Moreover, if $\mathcal{T}$ is countable, then $\bigcup \mathcal{T} \in \mathbb{P}_{\text {hom }}$.

Pr o of. Trivial. 
R e mark. The above lemma says that $\mathbb{P}_{\text {hom }}$ is $\omega_{1}$-closed, which means that every countable decreasing sequence in $\mathbb{P}_{\text {hom }}$ has a lower bound in $\mathbb{P}_{\text {hom }}$.

Lemma 3. Let $T \in \mathbb{P}_{\text {hom }}$ with $\mathrm{ht}(T)=\alpha$ for some limit ordinal $\alpha<\omega_{1}$. Let $\mathcal{C}$ be a countable subset of $\mathcal{B}(T)$. Then there exists $\bar{T} \in \mathbb{P}_{\text {hom }}$ such that $\bar{T}<T$ and for every $C \in \mathcal{C}, \bigcup C \in \bar{T}_{\alpha}$.

Proof. Without loss of generality, we can assume that for every $t \in T$, there exists $C \in \mathcal{C}$ such that $t \in C$. (This will guarantee the normality of the resulting tree.) We now construct inductively a sequence of countable trees $\left\langle T_{n}: n \in \omega\right\rangle$ such that:

(1) $T_{0}=T \cup\{\bigcup C: C \in \mathcal{C}\}$

(2) for every $n \in \omega, \operatorname{ht}\left(T_{n}\right)=\alpha+1$, and

(3) for every $n \in \omega$,

$$
T_{n+1}=T_{n} \cup\left\{F_{s, t}(u): s, t \in T_{n}, \operatorname{ht}(s)=\operatorname{ht}(t) \text { and } u \in\left(T_{n}\right)_{\alpha}\right\} .
$$

Note that if $I$ is an isomorphism from $T$ to $T$, then for every $B \in \mathcal{B}(T)$, $I[B] \in \mathcal{B}(T)$.

Let $\bar{T}=\bigcup_{n \in \omega} T_{n}$. It is obvious that $\bar{T}$ is countable and for any $s, t \in \bar{T}$ such that $h t(s)=\operatorname{ht}(t), F_{s, t}$ is an isomorphism from $\bar{T}$ to $\bar{T}$. Hence $\bar{T} \in \mathbb{P}_{\text {hom }}$, $\bar{T}<T$ and for every $C \in \mathcal{C}, \cup C \in T_{0} \subseteq \bar{T}$.

Next we discuss our forcing method. For the terminology and basic facts on forcing, see [K2] and [Je2]. We always assume the consistency of ZFC and let $M$ be a countable transitive model of ZFC. In the forcing arguments, we always let $\dot{a}$ be a name of $a$. For every element $a$ in the ground model, we may use $a$ itself as its name. Let $\mathbb{P}$ be a poset in a model $M, \dot{a}$ be a $\mathbb{P}$-name for $a$ and $G$ be a $\mathbb{P}$-generic filter over $M$. Then $\dot{a}_{G}$ is the value of $\dot{a}$ in $M[G]$ (see $\left[\mathrm{K} 2\right.$, p. 189] for the definition of $\dot{a}_{G}$ ).

Let $I, J$ be two sets. Let

$$
\operatorname{Fn}\left(I, J, \omega_{1}\right)=\left\{p: p \subseteq I \times J \text { is a function and }|p|<\omega_{1}\right\}
$$

be a poset ordered by reverse inclusion. Let $I$ be a subset of a cardinal $\kappa$. Let

$$
\begin{aligned}
\operatorname{Lv}\left(I, \omega_{1}\right)=\left\{p: p \subseteq\left(I \times \omega_{1}\right) \times \kappa \text { is a function, }|p|<\omega_{1}\right. \\
\text { and } \forall\langle\alpha, \beta\rangle \in \operatorname{dom}(p)(p(\alpha, \beta) \in \alpha)\}
\end{aligned}
$$

be a poset ordered by reverse inclusion. The poset $\operatorname{Lv}(\kappa, \mu)$ for some regular cardinals $\kappa>\mu$ is usually called a $<\mu$-support Lévy collapsing order. Let $T$ be a tree and $I$ be an index set. For a function $p$ from $I$ to $T$, let $\operatorname{supt}(p)$, the support of $p$, be the set $\{i \in I: p(i) \neq \emptyset\}$. Let

$$
\mathbb{P}\left(T, I, \omega_{1}\right)=\left\{p: p \in T^{I},|\operatorname{supt}(p)|<\omega_{1}\right\} .
$$


For any $p, p^{\prime} \in \mathbb{P}\left(T, I, \omega_{1}\right)$, define $p \leq p^{\prime}$ iff for every $i \in I, p^{\prime}(i) \leq_{T} p(i)$. Let $\mathbb{R}$ be a poset and $\dot{T}$ be an $\mathbb{R}$-name for a tree $T$. Let

$$
\mathbb{P}\left(\dot{T}, I, \omega_{1}\right)=\left\{\dot{q}: \dot{q} \in(\dot{T})^{I},|\operatorname{supt}(\dot{q})|<\omega_{1}\right\} .
$$

Then $\mathbb{P}\left(\dot{T}, I, \omega_{1}\right)$ is an $\mathbb{R}$-name for the poset $\mathbb{P}\left(T, I, \omega_{1}\right)$ if forcing with $\mathbb{R}$ adds no new countable sequence. Let $\mathbb{Q}=\mathbb{P}\left(T, I, \omega_{1}\right)$ (or $\left.\mathbb{P}\left(\dot{T}, I, \omega_{1}\right)\right)$ and $J \subseteq I$. We write $\mathbb{Q} \mid J$ for the set $\{p\lceil J: p \in \mathbb{Q}\}$. If $H$ is a $\mathbb{Q}$-generic filter, then let $H_{J}=\{p\lceil J: p \in H\}$.

LEMMA 4. Let $T$ be an $\omega_{1}$-tree and $\mathbb{P}$ be an $\omega_{1}$-closed poset in a model $M$. Let $G$ be a $\mathbb{P}$-generic filter over $M$. Assume that there exists a branch of $T$ in $M[G] \backslash M$. Then $T$ is neither a Kurepa tree nor a Jech-Kunen tree in $M$. Moreover, there exists an isomorphic embedding from $\left\langle 2^{<\omega_{1}}, \subseteq\right.$ into $T$.

Proof. See [K2, p. 260] and [Ju, Theorem 4.9].

Lemma 5. Let $M$ be a model, $\mathbb{P}=\left(\mathbb{P}_{\text {hom }}\right)^{M}$ and let $G$ be a $\mathbb{P}$-generic filter over $M$. Let $T_{G}=\bigcup G$. Then the generic tree $T_{G}$ is a homogeneous normal $\omega_{1}$-tree with every level countable. In fact, $T_{G}$ is a Suslin tree.

Proof. See [Je2, Theorem 48] for the proof. The homogeneity of $T_{G}$ follows from Lemma 2.

Lemma 6. Let $M$ be a model, $I$ be an index set in $M, \mathbb{P}=\left(\mathbb{P}_{\text {hom }}\right)^{M}, T_{\dot{G}}$ be a $\mathbb{P}$-name for the $\mathbb{P}$-generic tree $T_{G}$, and $\dot{\mathbb{Q}}=\mathbb{P}\left(T_{\dot{G}}, I, \omega_{1}\right)$, which is a $\mathbb{P}$-name for $\mathbb{P}\left(T_{G}, I, \omega_{1}\right)$. Let $G * H$ be a $\mathbb{P} * \dot{\mathbb{Q}}$-generic filter over $M$. Then $M^{\omega} \cap M[G * H] \subseteq M$.

Proof. Suppose that there is an $f \in M^{\omega} \cap M[G * H]$ such that $f \notin M$. Let $\langle p, \dot{q}\rangle \in \mathbb{P} * \dot{\mathbb{Q}}$ such that

$$
\langle p, \dot{q}\rangle \Vdash \dot{f} \in A^{\omega} \backslash M
$$

for some $A \in M$.

We now want to construct a sequence $\left\langle\left\langle p_{n}, \dot{q}_{n}\right\rangle \in \mathbb{P} * \dot{\mathbb{Q}}: n \in \omega\right\rangle$ in $M$ such that for every $n \in \omega$,

(1) $\left\langle p_{n+1}, \dot{q}_{n+1}\right\rangle \leq\left\langle p_{n}, \dot{q}_{n}\right\rangle \leq\langle p, \dot{q}\rangle$,

(2) $\exists a_{n} \in A\left(\left\langle p_{n}, \dot{q}_{n}\right\rangle \Vdash \dot{f}(n)=a_{n}\right)$,

(3) $\forall i \in \operatorname{supt}\left(\dot{q}_{n}\right) \exists t_{n}(i) \in p_{n} \backslash p_{n-1}\left(p_{n} \Vdash \dot{q}_{n}(i)=t_{n}(i)\right)$.

The contradiction follows from the construction. Let $\bar{p}_{\omega}=\bigcup_{n \in \omega} p_{n}$. For every $i \in \bigcup_{n \in \omega} \operatorname{supt}\left(\dot{q}_{n}\right)$, let

$$
C_{i}=\left\{t \in \bar{p}_{\omega}: \exists n \in \omega\left(t<t_{n}(i)\right)\right\} .
$$

By $(3), C_{i} \in \mathcal{B}\left(\bar{p}_{\omega}\right)$. By Lemma 3 , there is a $p_{\omega} \in \mathbb{P}, p_{\omega} \leq \bar{p}_{\omega}$, such that $\bigcup C_{i}$ $\in p_{\omega}$. Define $\dot{q}_{\omega}$ from $I$ to $T_{\dot{G}}$ such that $\dot{q}_{\omega}(i)=\bigcup C_{i}$ if $i \in \bigcup_{n \in \omega} \operatorname{supt}\left(\dot{q}_{n}\right)$ 
and $\dot{q}_{\omega}(i)=\emptyset$ otherwise. (In fact, $q$ is in $M$.) Then $\left\langle p_{\omega}, \dot{q}_{\omega}\right\rangle \leq\left\langle p_{n}, \dot{q}_{n}\right\rangle$ for every $n \in \omega$. Hence, for every $n \in \omega$,

$$
\left\langle p_{\omega}, \dot{q}_{\omega}\right\rangle \Vdash \dot{f}(n)=a_{n} .
$$

This contradicts $f \notin M$.

Assume that we have already had $\left\langle p_{n}, \dot{q}_{n}\right\rangle$ for every $n \leq m$.

First, let $\langle r, \dot{s}\rangle \leq\left\langle p_{m}, \dot{q}_{m}\right\rangle$ and $a_{m+1} \in A$ such that

$$
\langle r, \dot{s}\rangle \Vdash \dot{f}(m+1)=a_{m+1} .
$$

For every $i \in \operatorname{supt}(\dot{s})$,

$$
r \Vdash \exists \alpha \in \omega_{1}\left(\dot{s}(i) \in 2^{\alpha}\right) .
$$

Then there exist $\alpha \in \omega_{1}$ and $r^{\prime} \leq r$ such that

$$
r^{\prime} \Vdash \dot{s}(i) \in 2^{\alpha} .
$$

Since $\mathbb{P}$ is $\omega_{1}$-closed and

$$
r^{\prime} \Vdash \text { The domain of } \dot{s}(i) \text { is } \alpha \text {, a countable ordinal, }
$$

there exist $t(i) \in 2^{\alpha}$ and $r^{\prime \prime} \leq r^{\prime}$ such that

$$
r^{\prime \prime} \Vdash \dot{s}(i)=t(i) .
$$

Let $r^{\prime \prime \prime} \leq r^{\prime \prime}$ such that $\operatorname{ht}\left(r^{\prime \prime \prime}\right)>\max \left\{\alpha, \operatorname{ht}\left(p_{m}\right)\right\}$. Then

$$
r^{\prime \prime \prime} \Vdash \dot{s}(i)=t(i) \in r^{\prime \prime \prime}
$$

because $\Vdash \dot{s}(i) \in T_{\dot{G}}$.

Since $\operatorname{supt}(\dot{s})$ is countable and $\mathbb{P}$ is $\omega_{1}$-closed, we can find $p_{m+1} \leq r^{\prime \prime \prime}$ such that

$$
\forall i \in \operatorname{supt}(\dot{s}) \exists \alpha<\operatorname{ht}\left(p_{m+1}\right) \exists t(i) \in\left(p_{m+1}\right)_{\alpha}\left(p_{m+1} \Vdash \dot{s}(i)=t(i)\right) .
$$

Let $t_{m+1}(i) \in p_{m+1} \backslash p_{m}$ such that $t(i) \leq t_{m+1}(i)$ and define $\dot{q}_{m+1}(i)=$ $t_{m+1}(i)$ if $i \in \operatorname{supt}(\dot{s})$ and $\dot{q}_{m+1}(i)=\emptyset$ otherwise. This ends the construction and the sequence we have constructed does obviously satisfy (1)-(3).

Re mark. The poset $\mathbb{P} * \mathbb{Q}$ in Lemma 6 is, in fact, strategically complete. Let $\mathbb{R}$ be any poset. Two players, $I$ and $I I$, choose from $\mathbb{R}$ successively the members of a decreasing sequence

$$
a_{0} \geq b_{0} \geq a_{1} \geq b_{1} \geq \ldots \geq a_{n} \geq b_{n} \geq \ldots
$$

$I$ chooses the $a_{n}$ 's and $I I$ chooses the $b_{n}$ 's. II wins the game if and only if the sequence has a lower bound in $\mathbb{R}$. We call $\mathbb{R}$ strategically complete if $I I$ has a winning strategy. It has been shown that $\mathbb{R}$ is strategically complete if and only if there exists a poset $\mathbb{S}$ such that $\mathbb{R} \times \mathbb{S}$ has a dense subset which is $\omega_{1}$-closed (see [Je3, p. 90]). In our case, the poset $\mathbb{P} * \dot{\mathbb{Q}}$ has an $\omega_{1}$-closed dense subset. 
THEOREM 7. Assuming the existence of an inaccessible cardinal, it is consistent with $\mathrm{CH}$ plus $2^{\omega_{1}}>\omega_{2}$ that there exist Kurepa trees but there are no Jech-Kunen trees.

Proof. Let $M$ be a model of GCH, $\kappa$ be an inaccessible cardinal and $\lambda>\kappa$ be a regular cardinal in $M$. In $M$, let $\mathbb{P}_{1}=\operatorname{Lv}\left(\kappa, \omega_{1}\right), \mathbb{P}_{2}=\mathbb{P}_{\text {hom }}, T_{\dot{G}_{2}}$ be a $\mathbb{P}_{2}$-name for the $\mathbb{P}_{2}$-generic tree $T_{G_{2}}=\bigcup G_{2}$, where $G_{2}$ is a $\mathbb{P}_{2}$-generic filter, and $\dot{\mathbb{Q}}=\mathbb{P}\left(T_{\dot{G}_{2}}, \lambda, \omega_{1}\right)$. Let $G_{1} \times\left(G_{2} * H\right)$ be a $\mathbb{P}_{1} \times\left(\mathbb{P}_{2} * \dot{\mathbb{Q}}\right)$-generic filter over $M$. We will show that $M\left[G_{1} \times\left(G_{2} * H\right)\right]=M\left[G_{1}\right]\left[G_{2} * H\right]$ is the model we are looking for.

Claim 7.1. $M^{\omega} \cap M\left[G_{1}\right]\left[G_{2} * H\right] \subseteq M$.

Proof of Claim 7.1. By Lemma $6, M^{\omega} \cap M\left[G_{2} * H\right] \subseteq M$. This implies that $\mathbb{P}_{1}$ is still $\omega_{1}$-closed in $M\left[G_{2} * H\right]$. Hence $\left(M\left[G_{2} * H\right]\right)^{\omega} \cap$ $M\left[G_{2} * H\right]\left[G_{1}\right] \subseteq M\left[G_{2} * H\right]$. So for every $f \in M^{\omega} \cap M\left[G_{2} * H\right]\left[G_{1}\right], f$ is in $M\left[G_{2} * H\right]$ and hence, $f$ is in $M$. The claim is true because $M\left[G_{1}\right]\left[G_{2} * H\right]=$ $M\left[G_{2} * H\right]\left[G_{1}\right]$.

Claim 7.2. $\mathbb{P}_{1} \times\left(\mathbb{P}_{2} * \dot{\mathbb{Q}}\right)$ has the $\kappa$-c.c.

Proof of Claim 7.2. A poset $\mathbb{R}$ is called $\lambda$-centered for some regular cardinal $\lambda$ if for any subset $S \subseteq \mathbb{R}$ with $|S| \geq \lambda$, there exists $S^{\prime} \subseteq S,\left|S^{\prime}\right| \geq \lambda$, such that any two elements in $S^{\prime}$ are compatible. By a simple $\Delta$-system lemma argument, we can show that $\mathbb{P}_{1}$ is $\kappa$-centered. Since $\left|\mathbb{P}_{2}\right|=\omega_{1}$, we have $\left|T_{\dot{G}_{2}}\right| \leq\left(\left|\mathbb{P}_{2}\right|^{\omega_{1}}\right)^{\omega_{1}}=\omega_{2}$. Again by a simple $\Delta$-system lemma argument, we can show that $\mathbb{P}_{2} * \dot{\mathbb{Q}}$ is $\kappa$-centered. In fact, it is also $\omega_{3}$-centered. Hence $\mathbb{P}_{1} \times\left(\mathbb{P}_{2} * \dot{\mathbb{Q}}\right)$ is $\kappa$-centered, which implies the $\kappa$-c.c.

Rem ark. By Claims 7.1 and 7.2 and the fact that $M\left[G_{1}\right] \vDash\left[\mathrm{CH}+2^{\omega_{1}}=\right.$ $\left.\omega_{2}=\kappa\right]$, we know that $\omega_{1}$ and all the cardinals greater than or equal to $\kappa$ in $M$ are preserved in $M\left[G_{1}\right]\left[G_{2} * H\right]$. We also know that $M\left[G_{1}\right]\left[G_{2} * H\right] \vDash$ $\left[\mathrm{CH}+2^{\omega_{1}}=\lambda>\kappa\right]$.

Claim 7.3. $T_{G_{2}}$ is a Kurepa tree with $\lambda$-many branches in $M\left[G_{1}\right]\left[G_{2} * H\right]$.

Proof of Claim 7.3. It is obvious that $T_{G_{2}}$ is an $\omega_{1}$-tree with every level countable (in fact, it is a Suslin tree in $M\left[G_{2}\right]$ ). In $M\left[G_{1}\right]\left[G_{2}\right], \dot{\mathbb{Q}}_{G_{2}}=$ $\mathbb{P}\left(T_{G_{2}}, \lambda, \omega_{1}\right)$ is a countable support (note that no new countable sequences of ordinals are added) product of $\lambda$-many copies of $T_{G_{2}}$. Then forcing with $\mathbb{Q}$ will add at least $\lambda$-many new branches to $T_{G_{2}}$. Hence $\lambda \leq\left|\mathcal{B}\left(T_{G_{2}}\right)\right| \leq$ $2^{\omega_{1}}=\lambda$.

Claim 7.4. There are no Jech-Kunen trees in $M\left[G_{1}\right]\left[G_{2} * H\right]$.

Proof of Claim 7.4. Suppose that $T$ is a Jech-Kunen tree in $M\left[G_{1}\right]\left[G_{2} * H\right]$. Since $|T|=\omega_{1}$, there exists a cardinal $\theta<\kappa$ and a subset $I$ of $\lambda$ with $|I| \leq \omega_{2}$ such that $T \in M\left[G_{1}^{\prime}\right]\left[G_{2} * H_{I}\right]$, where $G_{1}^{\prime}=G_{1} \cap \operatorname{Lv}\left(\theta, \omega_{1}\right)$ 
and $H_{I}=H \cap \mathbb{Q} \mid I$. This is true because $\mathbb{P}_{1}$ has the $\kappa$-c.c. and $\mathbb{P}_{2} * \dot{\mathbb{Q}}$ has the $\omega_{3}$-c.c. In $M\left[G_{1}^{\prime}\right]\left[G_{2} * H_{I}\right], 2^{\omega_{1}}<\kappa$, so that there exists a branch $b$ of $T$ in $M\left[G_{1}\right]\left[G_{2} * H\right] \backslash M\left[G_{1}^{\prime}\right]\left[G_{2} * H_{I}\right]$. Since $\operatorname{Lv}\left(\kappa \backslash \theta, \omega_{1}\right)$ in $M$ is still $\omega_{1}$-closed in $M\left[G_{1}^{\prime}\right]\left[G_{2} * H_{I}\right]$ and $T$ is a Jech-Kunen tree in $M\left[G_{1}\right]\left[G_{2} * H\right]$, it follows from Lemma 4 that $b \notin M\left[G_{1}\right]\left[G_{2} * H_{I}\right]$.

Let $M^{\prime}=M\left[G_{1}\right]\left[G_{2} * H_{I}\right]$. We now work in $M^{\prime}$. In $M^{\prime}, \mathbb{Q} \vdash(\lambda \backslash I)$ has the $\omega_{1}$-c.c. Then there exists $J \subseteq \lambda \backslash I$ with $|J|=\omega_{1}$ in $M^{\prime}$ such that $b \in M^{\prime}\left[H_{J}\right]$. Let $r \in H_{J}$ be such that

$$
r \Vdash_{\mathbb{Q} \backslash J} \exists b \in \mathcal{B}(T) \backslash M^{\prime} .
$$

Since $T_{G_{2}}$ is homogeneous (here we use the homogeneity of the tree), we can assume that

$$
\Vdash_{\mathbb{Q} \vdash J} \exists b \in \mathcal{B}(T) \backslash M^{\prime} .
$$

By the maximal principle we can find a $\mathbb{Q} \mid J$-name $\dot{b}$ in $M^{\prime}$ such that

$$
\Vdash_{\mathbb{Q} \mid J} \dot{b} \in \mathcal{B}(T) \backslash M^{\prime} .
$$

Since $b \notin M^{\prime}$, it follows that in $M^{\prime}$, the sentence $\Phi(\mathbb{Q} \mid J, T, \dot{b})$ is true, where $\Phi(X, Y, Z)$ is

$$
\forall s \in X \exists s_{0}, s_{1} \leq s \exists \alpha \in \omega_{1} \exists t_{0}, t_{1} \in Y_{\alpha}, t_{0} \neq t_{1}\left(s_{i} \Vdash t_{i} \in Z\right) \text { for } i=0,1 .
$$

In $M\left[G_{1}\right]\left[G_{2} * H\right], T$ has fewer than $\lambda$-many branches, so there exists $\mu<\lambda$ such that $I \cup J \subseteq \mu$ and every branch of $T$ in $M\left[G_{1}\right]\left[G_{2} * H\right]$ is already in $M^{\prime}\left[H_{\mu \backslash I}\right]$. Let $J^{\prime} \subseteq \lambda \backslash \mu$ be such that $\left|J^{\prime}\right|=|J|$ and let $\pi$ be the natural isomorphism from $\mathbb{Q} \mid J$ to $\mathbb{Q}\left\lceil J^{\prime}\right.$. Then in $M^{\prime}$

$$
\Vdash_{\mathbb{Q} \mid J^{\prime}} \pi_{*}(\dot{b}) \in \mathcal{B}(T)
$$

is true and

$$
M^{\prime} \vDash \Phi\left(\mathbb{Q} \mid J^{\prime}, T, \pi_{*}(\dot{b})\right),
$$

where $\pi_{*}$ is the map from $\mathbb{Q} \mid J$-names to $\mathbb{Q} \mid J^{\prime}$-names induced by $\pi$ (see $[\mathrm{K} 2$, p. 222] for the definition of $\pi_{*}$ ).

SubClaim 7.4.1. $M^{\prime}\left[H_{\mu \backslash I}\right] \models\left[\Phi\left(\mathbb{Q} \mid J^{\prime}, T, \pi_{*}(\dot{b})\right)\right.$ and $\left.\Vdash_{\mathbb{Q} \mid J^{\prime}} \pi_{*}(\dot{b}) \in \mathcal{B}(T)\right]$.

Proof of Subclaim 7.4.1. Let $H_{J^{\prime}}$ be a $\mathbb{Q} \mid J^{\prime}$-generic filter over $M^{\prime}\left[H_{\mu \backslash I}\right]$. Then $H_{J^{\prime}}$ is also a $\mathbb{Q} \mid J^{\prime}$-generic filter over $M^{\prime}$. Hence in $M^{\prime}\left[H_{J^{\prime}}\right]$, $\left(\pi_{*}(\dot{b})\right)_{H_{J^{\prime}}} \in \mathcal{B}(T)$. If $s_{i} \in H_{J^{\prime}}$, then $t_{i} \in\left(\pi_{*}(\dot{b})\right)_{H_{J^{\prime}}}$ is also true in $M^{\prime}\left[H_{J^{\prime}}\right]$.

In $M^{\prime}\left[H_{J^{\prime}}\right]$, forcing with $\mathbb{Q} \uparrow(\mu \backslash I)$ will not change the truth of the above sentences. Hence in $M^{\prime}\left[H_{J^{\prime}}\right]\left[H_{\mu \backslash I}\right]=M^{\prime}\left[H_{\mu \backslash I}\right]\left[H_{J^{\prime}}\right],\left(\pi_{*}(\dot{b})\right)_{H_{J^{\prime}}} \in \mathcal{B}(T)$ and $t_{i} \in\left(\pi_{*}(\dot{b})\right)_{H_{J^{\prime}}}$ are also true. This implies that

$$
M^{\prime}\left[H_{\mu \backslash I}\right] \vDash\left[\Phi\left(\mathbb{Q} \mid J^{\prime}, T, \pi_{*}(\dot{b})\right) \text { and } \Vdash_{\mathbb{Q} \mid J^{\prime}} \pi_{*}(\dot{b}) \in \mathcal{B}(T)\right] .
$$

This ends the proof of Subclaim 7.4.1. 
Since forcing with $\mathbb{Q} \mid J^{\prime}$ will not add new branches of $T$, it follows that $B=\left(\pi_{*}(\dot{b})\right)_{H_{J^{\prime}}}$ is already in $M^{\prime}\left[H_{\mu \backslash I}\right]$. In $M^{\prime}\left[H_{\mu \backslash I}\right]$, let

$$
D=\left\{r \in \mathbb{Q}\left\lceil J^{\prime}: \exists t \notin B\left(r \Vdash_{\mathbb{Q} \mid J^{\prime}} t \in \pi_{*}(\dot{b})\right)\right\} .\right.
$$

Then $D$ is dense in $\mathbb{Q} \mid J^{\prime}$ because $\Phi\left(\mathbb{Q} \mid J^{\prime}, T, \pi_{*}(\dot{b})\right)$ is true in $M^{\prime}\left[H_{\mu \backslash I}\right]$. If $r_{0} \in D \cap H_{J^{\prime}}$, then $r_{0} \Vdash \pi_{*}(\dot{b}) \neq B$. This contradicts $\left(\pi_{*}(\dot{b})\right)_{H_{J^{\prime}}}=B$.

THEOREM 8. Assuming the existence of an inaccessible cardinal, it is consistent with $\mathrm{CH}$ plus $2^{\omega_{1}}=\omega_{4}$ that there only exist Kurepa trees with $\omega_{3}$-many branches.

Proof. Let us follow the notation of the proof of Theorem 7. Let $\lambda=\kappa^{+}$ in $M$. Let

$$
\mathbb{P}_{3}=\operatorname{Fn}\left(\kappa^{++}, 2, \omega_{1}\right)=\operatorname{Fn}\left(\omega_{4}, 2, \omega_{1}\right)
$$

in $M\left[G_{1}\right]\left[G_{2} * H\right]$ (note $\mathbb{P}_{3}$ is absolute with respect to $M$ and $M\left[G_{1}\right]\left[G_{2} * H\right]$ ). Let $G_{3}$ be a $\mathbb{P}_{3}$-generic filter over $M\left[G_{1}\right]\left[G_{2} * H\right]$. In $M\left[G_{1}\right]\left[G_{2} * H\right]\left[G_{3}\right]$, the number of branches of $T_{G_{2}}$ is $\lambda=\kappa_{+}=\omega_{3}$ by Lemma 4 .

Let $T$ be any $\omega_{1}$-tree in $M\left[G_{1}\right]\left[G_{2} * H\right]\left[G_{3}\right]$. Then there exists $K \subseteq$ $\kappa^{++}$with $|K|=\omega_{1}$ such that $T \in M\left[G_{1}\right]\left[G_{2} * H\right]\left[G_{3}^{\prime}\right]$, where $G_{3}^{\prime}=G_{3} \cap$ $\operatorname{Fn}\left(K, 2, \omega_{1}\right)$.

If $|\mathcal{B}(T)|=\omega_{4}$ in $M\left[G_{1}\right]\left[G_{2} * H\right]\left[G_{3}\right]$, then forcing with $\operatorname{Fn}\left(\kappa^{++} \backslash K, 2, \omega_{1}\right)$ will add new branches to $T$. This implies $T$ is not a Kurepa tree by Lemma 4 .

If $|\mathcal{B}(T)|=\omega_{2}$ in $M\left[G_{1}\right]\left[G_{2} * H\right]\left[G_{3}\right]$, then by Lemma $4, T$ is already a Jech-Kunen tree with $\omega_{2}$-many branches in $M\left[G_{1}\right]\left[G_{2} * H\right]\left[G_{3}^{\prime}\right]$. Without loss of generality we can assume that $K=\omega_{1}$. So

$M\left[G_{1}\right]\left[G_{2} * H\right]\left[G_{3}^{\prime}\right] \vDash$

"There exists a Jech-Kunen tree with $\omega_{2}$-many branches".

But

$$
M\left[G_{1}\right]\left[G_{2} * H\right]\left[G_{3}^{\prime}\right]=M\left[G_{3}^{\prime}\right]\left[G_{1}\right]\left[G_{2} * H\right]=\bar{M}\left[G_{1}\right]\left[G_{2} * H\right],
$$

where $\bar{M}=M\left[G_{3}^{\prime}\right]$. By the same proof of Theorem 7 , we can also show that there are no Jech-Kunen trees in $\bar{M}\left[G_{1}\right]\left[G_{2} * H\right]$, a contradiction.

\section{References}

[Je1] T. Jech, Trees, J. Symbolic Logic 36 (1971), 1-14.

[Je2] —, Set Theory, Academic Press, New York 1978.

[Je3] —, Multiple Forcing, Cambridge University Press, 1986.

[Ji1] R. Jin, Some independence results related to the Kurepa tree, Notre Dame J. Formal Logic 32 (1991), 448-457.

[Ji2] -, A model in which every Kurepa tree is thick, ibid. 33 (1992), 120-125.

[Ju] I. Juhász, Cardinal functions II, in: Handbook of Set-Theoretic Topology, K. Kunen and J. E. Vaughan (eds.), North-Holland, Amsterdam 1984, 63-110. 
[K1] K. Kunen, On the cardinality of compact spaces, Notices Amer. Math. Soc. 22 (1975), 212.

[K2] - Set Theory. An Introduction to Independence Proofs, North-Holland, Amsterdam 1980.

[S1] S. Shelah, Proper Forcing, Springer, 1982.

[S2] - , new version of Proper Forcing, to appear.

[SJ] S. Shelah and R. Jin, A model in which there are Jech-Kunen trees but there are no Kurepa trees, preprint.

[T] S. Todorčević, Trees and linearly ordered sets, in: Handbook of Set-Theoretic Topology, K. Kunen and J. E. Vaughan (eds.), North-Holland, Amsterdam 1984, 235-293.

INSTITUTE OF MATHEMATICS

THE HEBREW UNIVERSITY

JERUSALEM, ISRAEL

DEPARTMENT OF MATHEMATICS

RUTGERS UNIVERSITY

NEW BRUNSWICK, NEW JERSEY 08903

U.S.A.
DEPARTMENT OF MATHEMATICS UNIVERSITY OF CALIFORNIA BERKELEY, CALIFORNIA 94720 U.S.A. 